Digestive Endoscopy

\title{
Virtual chromoendoscopy improves the diagnostic yield of small bowel capsule endoscopy in obscure gastrointestinal bleeding
}

\author{
Pedro Boal Carvalho ${ }^{\mathrm{a}, *}$, Joana Magalhães ${ }^{\mathrm{a}}$, Francisca Dias de Castro ${ }^{\mathrm{a}}$, \\ Tiago Cúrdia Gonçalves ${ }^{a}$, Bruno Rosa ${ }^{a}$, Maria João Moreira ${ }^{a}$, José Cotter ${ }^{\mathrm{a}, \mathrm{b}, \mathrm{c}}$ \\ a Alto Ave Hospital, Guimarães, Portugal \\ ${ }^{\mathrm{b}}$ Life and Health Sciences Research Institute (ICVS), School of Health Sciences, University of Minho, Braga, Portugal \\ ' ICVS/3B's, PT Government Associate Laboratory, Guimarães/Braga, Portugal
}

\section{A R T I C L E I N F O}

\section{Article history:}

Received 1 June 2015

Accepted 15 November 2015

Available online 23 November 2015

\section{Keywords:}

Capsule endoscopy

Obscure gastrointestinal bleeding

Small bowel diseases

Virtual chromoendoscopy

\begin{abstract}
A B S T R A C T
Background: Small bowel capsule endoscopy represents the initial investigation for obscure gastrointestinal bleeding. Flexible spectral imaging colour enhancement (FICE) is a virtual chromoendoscopy technique designed to enhance mucosal lesions, available in different settings according to light wavelength - FICE1, 2 and 3.

Aims: To compare the diagnostic yield of FICE1 and white light during capsule endoscopy in patients with obscure gastrointestinal bleeding.

Methods: Retrospective single-centre study including 60 consecutive patients referred for small bowel capsule endoscopy for obscure gastrointestinal bleeding. Endoscopies were independently reviewed in FICE1 and white light; findings were then reviewed by another researcher, establishing a gold standard. Diagnostic yield was defined as the presence of lesions with high bleeding potential (P2) angioectasias, ulcers or tumours.

Results: Diagnostic yield using FICE1 was significantly higher than white light (55\% vs 42\%, $p=0.021$ ). A superior number of P2 lesions was detected with FICE1 (74 vs 44, $p=0.003$ ), particularly angioectasias (54 vs 26, $p=0.002$ ), but not ulcers or tumours.

Conclusions: FICE1 was significantly superior to white light, resulting in a $13 \%$ improvement in diagnostic yield, and potentially bleeding lesions particularly angioectasias were more often observed. Our results support the use of FICE1 while reviewing small bowel capsule endoscopy for obscure gastrointestinal bleeding.
\end{abstract}

(c) 2015 Editrice Gastroenterologica Italiana S.r.l. Published by Elsevier Ltd. All rights reserved.

\section{Introduction}

Small bowel capsule endoscopy (SBCE) was introduced in clinical practice at the start of the millennium, allowing for the non-invasive observation of the entire small bowel mucosa, and facilitating the diagnosis of small bowel diseases [1]. Obscure gastrointestinal bleeding (OGIB), defined as bleeding of unknown origin that persists or recurs after a negative primary endoscopic study - esophagogastroduodenoscopy (EGD) and colonoscopy - is, alongside inflammatory bowel disease, one of the prime indications for SBCE $[1,2]$.

\footnotetext{
* Corresponding author at: Centro Hospitalar do Alto Ave - Guimarães, Rua dos Cutileiros, Creixomil, 4831-044 Guimarães, Portugal.

E-mail address: Pedroboalcarvalho@chaa.min-saude.pt (P. Boal Carvalho).
}

The diagnostic yield of SBCE in patients presenting with OGIB was previously shown to be superior to other modalities, including push enteroscopy, computed tomography and angiography [3,4], and non-inferior to double-balloon enteroscopy [5]. SBCE diagnostic capabilities, coupled with its non-invasiveness, helped cement its position as the first line diagnostic procedure in OGIB [1]. Nevertheless, despite the technological improvements such as increased battery time and improved image resolution, the diagnostic yield for white light SBCE in patients presenting with OGIB is $40-60 \%$ $[1,6]$.

Virtual chromoendoscopy techniques, such as the flexible spectral imaging colour enhancement (FICE, Fujinon Corporation ${ }^{\circledR}$, Saitama, Japan), were recently developed with the objective of enhancing surface patterns or mucosal lesions through the narrowing of white light bandwidth and reconstituting virtual images for different wavelengths of red, green and blue [7]. In FICE, different wavelength settings correspond to one of three different modes: 
FICE1 (wavelength red $595 \mathrm{~nm}$, green $540 \mathrm{~nm}$, blue $535 \mathrm{~nm}$ ), FICE2 (wavelength red $420 \mathrm{~nm}$, green $520 \mathrm{~nm}$, blue $530 \mathrm{~nm}$ ), or FICE3 (wavelength red $595 \mathrm{~nm}$, green $570 \mathrm{~nm}$, blue $415 \mathrm{~nm}$ ). FICE has been employed in both the upper and lower digestive tract $[8,9]$, as well as in the small bowel, through double balloon enteroscopy [10]. The use of FICE in SBCE was demonstrated by several authors to be both feasible and simple to use, as well as easily accessible, as it is integrated in the RAPID Reader workstation (Given Imaging ${ }^{\circledR}$, Yoqneam, Israel) [6,7]. Some authors reported an improvement in small bowel lesions visibility when using FICE [11,12], but the impact on the diagnostic yield for OGIB is yet unclear $[6,13,14]$. FICE1, in particular, was shown by some authors to be superior to other virtual chromoendoscopy modes during SBCE reviewing $[11,15,16]$.

We aimed to compare diagnostic yield and number of potentially bleeding small bowel lesions between white light (WL) and FICE1 in patients undergoing SBCE for OGIB. Furthermore, we compared the incidence of rebleeding at 24 months for patients with confirmed negative examinations vs patients with negative examinations under WL but having P2 lesions detected by FICE.

\section{Methods}

We performed a retrospective single-centre study including 60 consecutive patients presenting with OGIB who underwent SBCE in our department starting January 1st, 2011 (last patient included on May 11th, 2011). In every patient, an EGD and colonoscopy were performed, prior to the SBCE (interval $<6$ months), which were non-diagnostic. OGIB was classified as visible when the patient presented with either melena or haematochezia, and occult if there was iron deficient anaemia (haemoglobin $<13 \mathrm{~g} / \mathrm{dL}$ for men, $<12 \mathrm{~g} / \mathrm{dL}$ for women) or a positive faecal occult blood test (FOBT). All patients had written informed consent for SBCE examination. The study was previously approved by the Hospital ethics committee.

Patient drug use and relevant medical history was registered on a checklist prior to the SBCE procedure, per department protocol. Past medical history was collected for diabetes mellitus, arterial hypertension, chronic kidney disease (defined as a creatinine clearance rate $<60 \mathrm{~mL} / \mathrm{min} / \mathrm{m}^{2}$ ) and ischaemic heart disease, as well as drug use in the 30 days previous to the SBCE: antiplatelet drugs (low-dose aspirin, thienopyridines), anticoagulant drugs [warfarin, low molecular weight heparin (LMWH)] and non-steroidal antiinflammatory drugs (NSAIDs). Patients with incomplete SBCE, defined as the capsule not reaching the cecum before the battery expired, as well as patients with less than 24 months of followup, were excluded. Patients submitted to therapy of small bowel lesions found on SBCE were excluded. PillCam ${ }^{\text {TM }}$ SB2 capsule (Given Imaging ${ }^{\circledR}$, Yoqneam, Israel) was used. Patients were instructed to adhere to a clear liquid diet on the day prior to the procedure, as well as a $12 \mathrm{~h}$ fast; no additional bowel preparation was employed.

Four gastroenterologists with experience in SBCE (>100 examinations), whose inter-observer percentage of agreement was previously shown to be excellent (Fleiss Kappa coefficient $>0.8$ for FICE1) [16], independently reviewed the SBCE as follows: each one reviewed 15 videos with conventional WL and another 15 videos using FICE1, such that each SBCE was reviewed once with WL by one investigator and once with FICE1 by another investigator, avoiding any overlap, and blinded to the other researchers' findings. All procedures were reviewed with a reading speed of 10-12 frames per second.

All SBCE were afterwards independently reviewed by another gastroenterologist with vast experience in SBCE (>500 examinations), in order to establish a gold standard and identify false positive results from either visualization technique. Small bowel lesions were described using the extensively employed
Table 1

Baseline characteristics of patients who underwent small bowel capsule endoscopy for obscure gastrointestinal bleeding.

\begin{tabular}{lc}
\hline$N$ & 60 \\
Median age (years) & $63.8(19-89)$ \\
Female gender & $36(60.0 \%)$ \\
Occult OGIB & $48(80 \%)$ \\
Visible OGIB & $12(20 \%)$ \\
Median haemoglobin (g/dL) & $9.0(5.0-15.0)$ \\
Diabetes mellitus & $24(40.0 \%)$ \\
Chronic kidney disease & $5(8.3 \%)$ \\
Arterial hypertension & $30(50.0 \%)$ \\
Ischaemic heart disease & $17(28.3 \%)$ \\
Antiplatelet drugs & $31(51.7 \%)$ \\
Anticoagulant drugs & $14(23.3 \%)$ \\
NSAIDs & $12(20.0 \%)$ \\
\hline
\end{tabular}

OGIB, obscure gastrointestinal bleeding; NSAID, non-steroidal anti-inflammatory drugs.

classification of Saurin et al. [17], as P0 (no bleeding potential, such as nodules and lymphangiectasias), P1 (uncertain bleeding potential, such as red spots or small erosions) and P2 (high bleeding potential, such as angioectasias, ulcers, tumours or varices). Primary outcomes were defined as the number of P2 lesions encountered and the diagnostic yield using FICE1 and WL. Diagnostic yield was considered for the presence of at least one P2 lesion in the small bowel after false positive result exclusion.

The secondary outcome was to analyze rebleeding rates within two years of SBCE. Rebleeding was defined as evidence of melena or haematochezia, symptomatic anaemia, need for blood transfusion or a decrease in haemoglobin concentration value superior to $2 \mathrm{~g} / \mathrm{dL}$, following non-diagnostic EGD and total colonoscopy.

Statistical analysis was performed using SPSS $21.1^{\mathrm{TM}}$ (WinWrap Basics $^{\mathrm{TM}}$ ). Univariate analysis was performed for comparison of findings, diagnostic yield and rebleeding rate between FICE1 and WL, using paired-samples $t$ test for continuous variables and the McNemar test for categorical variables. Statistical significance was defined for $p$-value $<0.05$. Quantitative values are presented as mean \pm standard deviation.

\section{Results}

Overall 60 patients were enrolled (60\% females, mean age, $64 \pm 18$ years); 12 were inpatients (20\%), and bleeding site was visible in 12 cases (20\%); mean haemoglobin concentration values were $9.0 \pm 2.2 \mathrm{~g} / \mathrm{dL}$. Forty five patients (75\%) presented with at least one comorbidity. The population baseline characteristics are summarized in Table 1.

During FICE1 visualization, 63 angioectasias, 17 ulcers and 4 tumours were identified. After video review and comparison, 9 of the angioectasias and 1 tumour were found to correspond to false positive results (considered by the central reader to be either an artefact or a P1 lesion, such as a red spot), and were subsequently excluded from the comparative analysis. The false positive rate for P2 lesions during FICE1 observation was 10/84(11.9\%), corresponding to a specificity of $88 \%$, while no false positive result for P2 lesions was obtained in WL visualization. SBCE findings for both FICE1 and WL after the exclusion of false positives are summarized in Table 2. No false negatives were found during FICE1 SBCE reviewing.

Diagnostic yield for SBCE in patients presenting with OGIB was significantly higher when the procedures were reviewed in FICE1 compared to $\mathrm{WL}$ ( $55 \%$ vs $42 \%, p=0.021$ ); 8 patients previously considered to have a normal exam or P1 lesions during WL visualization were diagnosed with P2 lesions with FICE1, corresponding to a $13 \%$ increase of the SBCE diagnostic yield. Moreover, the total number of P2 lesions observed with FICE1 was superior when WL was employed ( 74 vs $44, p=0.003$ ). 
Table 2

Small bowel capsule endoscopy findings in white light and flexible spectral imaging colour enhancement mode 1.

\begin{tabular}{llrr}
\hline & White light & FICE1 & \multicolumn{1}{c}{$p$} \\
\hline Bleeding risk, patients (\%) & & & \\
P0 & $18(30)$ & $9(15)$ & 0.021 \\
P1 & $17(28)$ & $18(30)$ & \\
P2 & $25(42)$ & $33(55)$ & \\
Small bowel capsule endoscopy lesions & & \\
$\quad$ P1 lesions & & & \\
$\quad$ Erosions/red spots & 43 & 73 & $<0.001$ \\
P2 lesions & & 54 & 0.003 \\
$\quad$ Angioectasias & 26 & 17 & 0.548 \\
$\quad$ Ulcers & 15 & 3 & 1.000 \\
$\quad$ Tumours & 3 & & \\
\hline
\end{tabular}

FICE1, flexible spectral imaging colour enhancement mode 1; P0, lesions with no bleeding potential; P1, lesions with uncertain bleeding potential; P3, lesions with high bleeding potential.

When analysing the different P2 lesions, significantly more small bowel angioectasias were found when using FICE1 (54 vs 26, $p=0.003$ ), but no differences were observed regarding ulcers ( $17 \mathrm{vs}$ $15, p=0.568$ ) or tumours ( 3 vs $3, p=1.000$ ). Visualization of a small angioectasia during SBCE using FICE1 and WL is presented in Fig. 1.

With regard to non-P2 small bowel lesions, FICE1 allowed for the observation of a significantly larger number of erosions (73 vs $43, p<0.001$ ) after false-positive results were excluded. The rate for false positive P1 lesions was 12/93 (12.9\%) during FICE1 observation, and 3/46 (6.5\%) for WL visualization.

Rebleeding during follow-up was observed in 16 patients (26.7\%), of which 10 during the first year (61.7\%). Patients with at least one P2 lesion on FICE1 experienced rebleeding during follow-up in $11 / 33$ cases (33.3\%), compared to 5/27 patients (18.5\%) with no P2 lesions ( $p=0.197)$. FICE1 detected P2 lesions in $11 / 16$ patients presenting with rebleeding (68.7\%), compared to $8 / 16$ for WL visualization $(50.0 \% ; p=0.250)$. No differences were observed in the mean detected P2 lesions during SBCE in patients presenting with rebleeding ( 1.38 vs $1.18, p=0.709$ ). Patients' gender, age, past medical history, current medication, haemoglobin levels and OGIB presentation were not related to either outcome.

\section{Discussion}

Despite being used in SBCE reviewing for almost a decade, the results for the usefulness of virtual chromoendoscopy in diagnostic improvement are yet conflicting.

Pioneering studies, such as the one developed by Pohl et al. [7], reported an enhancement of the hypervascularity as well the contrasting mucosal patterns when using FICE for reviewing SBCE videos. Further studies $[11,13,18]$, analysing isolated SBCE frames, supported the benefits of FICE modes 1 and 2 for improving the detectability of angioectasias and erosions/ulcers.

Our department conducted a study [16] evaluating 100 small bowel lesions detected by SBCE, including mucosal breaks such as ulcers or erosions, vascular lesions such as angioectasias and villous pattern abnormalities such as oedema or atrophy. The authors observed an overall improvement of delineation of small bowel mucosal lesions with FICE, particularly with FICE1: the delineation of the lesions was improved in $77 \%$ of the cases, with a percentage of agreement between investigators of $89 \%$, compared to an improvement of $74 \%$ and $41 \%$ for FICE modes 2 and 3 [16]. The advantages of FICE1 compared to other modes were further substantiated by Krystallis et al. [19] and Imagawa et al. [20].

The advantages of FICE for the review of the entire SBCE videos in the clinical setting of patients with OGIB are not as clear. The virtual chromoendoscopy technique implies a learning curve [6,7], as
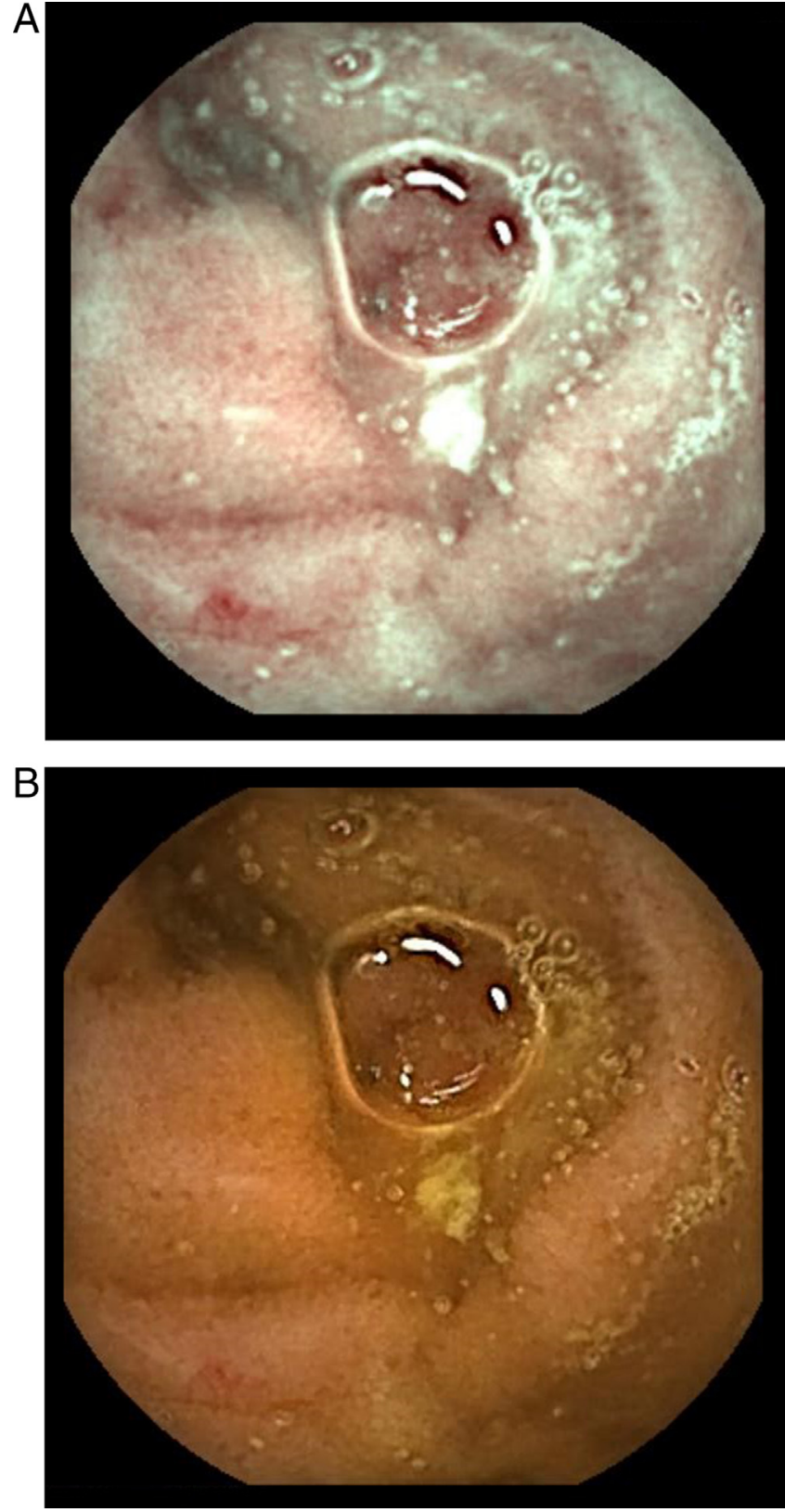

Fig. 1. Angioectasia visualization during small bowel capsule endoscopy using FICE1 (Panel A) and white light visualization (Panel B).

non-pathological lesions such as exuberant but normal vasculature or prominent folds may be mistaken for small bowel angioectasias owning to changes in the colour patterns, hindering SBCE reviewing.

Gupta et al. [6] found no increase in diagnostic yield or number of P2 lesions when FICE was used (153 vs $118, p=0.15$ ), Duque et al. [21] encountered exclusively more erosions with FICE2 than WL, with no differences in other lesions. In contrast, in a study by Kobayashi et al. [15], FICE1 detected significantly more angioectasias and ulcers than WL, while no benefits were found for FICE modes 2 or 3, and other authors found FICE1 to be superior to WL for the detection of both P1 and P2 lesions [12,13]. In another recent work by Sakai et al. [14], not only did FICE1 improve detectability of angioectasias, erosions and ulcers compared to WL, but crucially, its diagnostic yield was not affected by the presence of luminal bile, unlike WL and FICE2; these results were further explained as 
FICE1 seemingly produces the virtual effect of transparentizing bile [13].

In our study, we observed not only an increase in the number of P2 lesions (74 vs 44, $p=0.003$ ), but also a significant increase on the diagnostic yield for SBCE when FICE1 was used (55\% vs $42 \%, p=0.021$ ), which to our knowledge has not been previously reported in the literature. These results may be explained by the following: firstly, in our study, we defined diagnostic yield for the presence of P2 lesions, as not only are they associated with a higher risk of bleeding $[17,22]$, but also because up to $10 \%$ of healthy subjects may present with clinically silent erosions (P1 lesions) on SBCE [23]; secondly, we have had significant previous experience with the use of FICE in SBCE [16,24]; thirdly, we included a large series with more patients than previous works; and fourthly, we employed paired samples analysis, allowing for the exclusion of external variables, as well as limiting the comparison to WL vs FICE1.

In our study, we encountered an overall lower diagnostic yield than reported in the literature [1], but such results are justified by the restrictive inclusion of exclusively P2 lesions when accounting for diagnostic criteria.

A significantly superior number of angioectasias was observed when using FICE1 (54 vs 26, $p=0.003$ ), but no differences were found regarding ulcers or tumours. The benefits of FICE mode for identifying angioectasias were previously reported by Nakamura et al. [25] and Sakai et al. [14], among others, and relates to the heightening of the vascular arborization $[6,7]$. Some authors reported an increase in the detection rates of erosions/ulcers [12,18] when using FICE, contrasting with our findings. Unlike the previous authors, we analyzed erosions and ulcers separately, and despite not observing more ulcers with FICE1 (15 vs 17, $p=0.568$ ), we did find more erosions (73 vs $43, p<0.001$ ) when reviewing the SBCE in FICE1, which suggests that ulcers are already easier to detect than erosions, and therefore less likely to be missed during white light visualization.

In our work, no differences were found in the detection of small bowel tumours; notwithstanding the fact that a small number of such lesions were observed, and therefore allowing for Type II errors, these results are consistent with the ones reported by the majority of the published data $[13,15,20,25]$.

Finally, rebleeding was encountered in 16 patients (26.7\%) during a two-year period. In our series, the presence of P2 lesions, as well as their number, was not significantly associated with an increased risk of rebleeding. However, we encountered rebleeding in 11/33 of the patients presenting with at least one P2 lesion in FICE1 compared to 5/27 in patients with negative SBCE. Additionally, in this specific high risk population, FICE1 allowed for the detection of P2 lesions in 3 additional patients compared to white light visualization ( 11 vs $8, p=0.250$ ). The prognostic relevance of FICE in patients presenting with rebleeding was demonstrated in a recently published study by Dias de Castro et al. [24], where more than $80 \%$ of patients presenting with rebleeding after a negative SBCE performed for OGIB and observed in WL, were found to have potentially bleeding lesions when visualized using FICE1. Further studies with a larger sample sizes are warranted to confirm the likely prognostic importance of FICE1 when analysing SBCE for P2 lesions, namely regarding the potential therapeutic management of such lesions.

In conclusion, FICE1 was associated with an improved diagnostic yield, as well as the detection of a superior number of P2 lesions, namely angioectasias. These results, together with the fact that false positive results can be readily identified and rectified, support the systematic use of FICE1 when reviewing SBCE performed for OGIB.

\section{Conflict of interest}

None declared.

\section{References}

[1] Ladas SD, Triantafyllou K, Spada C, et al. European Society of Gastrointestinal Endoscopy (ESGE): recommendations (2009) on clinical use of video capsule endoscopy to investigate small-bowel, esophageal and colonic diseases. Endoscopy 2010;42:220-7.

[2] Raju GS, Gerson L, Das A, et al. American Gastroenterological Association (AGA) Institute medical position statement on obscure gastrointestinal bleeding. Gastroenterology 2007;133:1694-6.

[3] Triester SL, Leighton JA, Leontiadis GI, et al. A meta-analysis of the yield of capsule endoscopy compared to other diagnostic modalities in patients with obscure gastrointestinal bleeding. American Journal of Gastroenterology 2005; 100:2407-18.

[4] Saperas E, Dot J, Videla S, et al. Capsule endoscopy versus computed tomographic or standard angiography for the diagnosis of obscure gastrointestinal bleeding. American Journal of Gastroenterology 2007;102:731-7.

[5] Pasha SF, Leighton JA, Das A, et al. Double-balloon enteroscopy and capsule endoscopy have comparable diagnostic yield in small-bowel disease: a metaanalysis. Clinical Gastroenterology and Hepatology 2008;6:671-6.

[6] Gupta T, Ibrahim M, Deviere J, et al. Evaluation of Fujinon intelligent chromo endoscopy-assisted capsule endoscopy in patients with obscure gastroenterology bleeding. World Journal of Gastroenterology 2011;17:4590-5.

[7] Pohl J, Aschmoneit I, Schuhmann S, et al. Computed image modification for enhancement of small-bowel surface structures at video capsule endoscopy. Endoscopy 2010;42:490-2.

[8] Pohl J, Nguyen-Tat M, Pech O, et al. Computed virtual chromoendoscopy for classification of small colorectal lesions: a prospective comparative study. American Journal of Gastroenterology 2008;103:562-9.

[9] Pohl J, May A, Rabenstein T, et al. Comparison of computed virtual chromoendoscopy and conventional chromoendoscopy with acetic acid for detection of neoplasia in Barrett's esophagus. Endoscopy 2007;39:594-8.

[10] Neumann H, Fry LC, Bellutti M, et al. Double-balloon enteroscopy-assisted virtual chromoendoscopy for small-bowel disorders: a case series. Endoscopy 2009:41:468-71.

[11] Imagawa $\mathrm{H}$, Oka $\mathrm{S}$, Tanaka $\mathrm{S}$, et al. Improved visibility of lesions of the small intestine via capsule endoscopy with computed virtual chromoendoscopy. Gastrointestinal Endoscopy 2011;73:299-306.

[12] Matsumura T, Arai M, Sato T, et al. Efficacy of computed image modification of capsule endoscopy in patients with obscure gastrointestinal bleeding. World Journal of Gastrointestinal Endoscopy 2012;4:421-8.

[13] Konishi M, Shibuya T, Mori H, et al. Usefulness of flexible spectral imaging color enhancement for the detection and diagnosis of small intestinal lesions found by capsule endoscopy. Scandinavian Journal of Gastroenterology 2014;49:501-5.

[14] Sakai E, Endo H, Kato S, et al. Capsule endoscopy with flexible spectral imaging color enhancement reduces the bile pigment effect and improves the detectability of small bowel lesions. BMC Gastroenterology 2012;12:83.

[15] Kobayashi Y, Watabe H, Yamada A, et al. Efficacy of flexible spectral imaging color enhancement on the detection of small intestinal diseases by capsule endoscopy. Journal of Digestive Diseases 2012;13:614-20.

[16] Cotter J, Magalhaes J, de Castro FD, et al. Virtual chromoendoscopy in small bowel capsule endoscopy: new light or a cast of shadow? World Journal of Gastrointestinal Endoscopy 2014;6:359-65.

[17] Saurin JC, Delvaux M, Gaudin JL, et al. Diagnostic value of endoscopic capsule in patients with obscure digestive bleeding: blinded comparison with video push-enteroscopy. Endoscopy 2003;35:576-84

[18] Nogales Rincon O, Merino Rodriguez B, Gonzalez Asanza C, et al. Utility of capsule endoscopy with flexible spectral imaging color enhancement in the diagnosis of small bowel lesions. Gastroenterologia y Hepatologia 2013;36:63-8

[19] Krystallis C, Koulaouzidis A, Douglas S, et al. Chromoendoscopy in small bowel capsule endoscopy: blue mode or Fuji Intelligent Colour Enhancement? Digestive and Liver Disease 2011;43:953-7.

[20] Imagawa H, Oka S, Tanaka S, et al. Improved detectability of small-bowel lesions via capsule endoscopy with computed virtual chromoendoscopy: a pilot study. Scandinavian Journal of Gastroenterology 2011:46:1133-7.

[21] Duque G, Almeida N, Figueiredo P, et al. Virtual chromoendoscopy can be a useful software tool in capsule endoscopy. Revista Espanola de Enfermedades Digestivas 2012;104:231-6.

[22] Curdia Goncalves T, Dias de Castro F, Moreira MJ, et al. Small bowel capsule endoscopy in obscure gastrointestinal bleeding: normalcy is not reassuring. European Journal of Gastroenterology and Hepatology 2014;26:927-32.

[23] Graham DY, Chan FK. Endoscopic ulcers with low-dose aspirin and reality testing. Gastroenterology 2005;128:807, author reply - 8 .

[24] Dias de Castro F, Magalhaes J, Boal Carvalho P, et al. Improving diagnostic yield in obscure gastrointestinal bleeding - how virtual chromoendoscopy may be the answer. European Journal of Gastroenterology and Hepatology 2015;27:735-40

[25] Nakamura M, Ohmiya N, Miyahara R, et al. Usefulness of flexible spectral imaging color enhancement (FICE) for the detection of angiodysplasia in the preview of capsule endoscopy. Hepato-Gastroenterology 2012;59:1474-7. 